\title{
La teleconsulta en enfermedades respiratorias durante la epidemia de COVID-19: a propósito de un caso
}

\author{
Remote consultation in respiratory diseases during the \\ COVID-19 epidemic: A case report
}

John Carlos Pedrozo-Pupo', Pablo Salomón Montes-Arcón², Adalberto Campo-Arias ${ }^{3}$

Fecha correspondencia:

Recibido: junio 12 de 2020. Revisado: agosto 28 de 2020.

Aceptado: septiembre 4 de 2020.

Forma de citar:

Pedrozo-Pupo JC, Montes-Arcón

PS, Campo-Arias A. La teleconsulta en enfermedades respiratorias durante la epidemia de COVID-19: a propósito de un caso. Rev CES Med. 2020; Especial COVID-19: 111-116.

Open access

(c) Derecho de autor

Licencia creative commons

Ética de publicaciones

Revisión por pares

Gestión por Open Journal System DOl: http://dx.doi.org/10.21615/ cesmedicina.34.COVID-19.15

ISSN 0120-8705

e-ISSN 2215-9177
Comparte

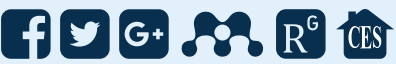

\begin{abstract}
Resumen
La pandemia de la enfermedad por coronavirus (COVID-19) trajo retos en la atención en salud e hizo de la teleconsulta una alternativa para la continuidad de la atención de los pacientes. La teleconsulta tiene ventajas, sin embargo, se deben considerar algunas limitaciones. Se presenta el caso de una mujer de 18 años con síntomas respiratorios de tres meses de evolución quien ameritó manejo en unidad de cuidados intensivos. Durante el confinamiento presentó síntomas respiratorios agudos sin deterioro importante en el estado general. En la teleconsulta, con la asistencia de un médico, el único hallazgo positivo fue la auscultación de un estridor laríngeo que orientó el manejo y diagnóstico final del caso y muestra algunas ventajas y limitaciones de la telemedicina en tiempos de crisis y posiblemente en tiempos regulares.
\end{abstract}

Palabras clave: Consulta remota; Enfermedades respiratorias; Informe de casos.

\begin{abstract}
The coronavirus disease pandemic (COVID-19) brought challenges to health care and made teleconsultation an alternative for the continuity of patient care. There are advantages to teleconsultation, but some limitations must be considered. We present the case of an 18 year-old woman with respiratory symptoms of three months of evolution who deserved to be managed in an intensive care unit. During the confinement she presented acute respiratory symptoms without significant deterioration in her general condition. In the teleconsultation, with the assistance of a physician, the only positive finding was the auscultation of a laryngeal stridor that guided the management and final diagnosis of the case and shows some advantages and limitations of telemedicine in times of crisis and possibly in regular times.
\end{abstract}

Keywords: Remote consultation; Respiratory tract diseases; Case reports.

\section{Introducción}

En el contexto mundial la pandemia de la enfermedad por coronavirus 


\author{
Sobre los autores: \\ 1. Médico neumólogo, \\ profesor Universidad del \\ Magdalena, Santa Marta, \\ Colombia. \\ 2. Médico, ESE Hospital César \\ Uribe Piedrahita, Caucasia, \\ Colombia. \\ 3. Médico Psiquiatra, \\ Epidemiólogo, Magíster en \\ salud sexual y reproductiva, \\ Profesor Universidad del \\ Magdalena.
}

La Organización Mundial de la Salud plantea tres ventajas de la teleconsulta: reducir el riesgo de infección, proteger a las personas de alto riesgo y la solidaridad tecnológica con otras regiones con menos acceso a la Medicina presencial.
(COVID-19) trajo consigo nuevos retos en la atención de los pacientes. Como medida para evitar el contagio masivo el aislamiento preventivo representa un escenario clínico distinto y con ello se hace necesario implementar estrategias para garantizar el acceso a los servicios de salud. Una de estas estrategias es la teleconsulta (1).

La teleconsulta se considera una importante herramienta que permite la continuidad de la atención de pacientes en situación de cronicidad y reducir el riesgo de infección por COVID-19 en pacientes y médicos en una consulta presencial (2). Asimismo, la teleconsulta puede ayudar a evitar el represamiento y mejorar la oportunidad de atención en los servicios de salud. Actualmente, alrededor mundo, se asumió la alternativa de la teleconsulta, donde está disponible, para evitar el riesgo de infección de los pacientes que necesitan un servicio de salud y obviamente del personal que está en contacto con ellos (3).

El avance tecnológico brinda unas oportunidades únicas y soluciones innovadoras que permiten al paciente consultar a un proveedor de atención médica a través de teleconferencias en tiempo real $(4,5)$.

La Organización Mundial de la Salud plantea tres ventajas de la teleconsulta: reducir el riesgo de infección, proteger a las personas de alto riesgo y la solidaridad tecnológica con otras regiones con menos acceso a la Medicina presencial (6). Además, la teleconsulta tiene el beneficio para la práctica médica de los profesionales, dado que disminuye su riesgo de exposición a la enfermedad, así como a la carga física, mental y emocional inherente a la atención convencional de pacientes durante la contingencia (7).

El objetivo es presentar un caso de una paciente en quien, durante el tiempo de pandemia, se hizo diagnóstico mediante teleconsulta asistida por un programa de videollamada.

\section{Resumen del caso}

Mujer de 18 años previamente sana quien consultó inicialmente al servicio de urgencias de una clínica privada de la ciudad al manifestar "mucha dificultad para respirar". El episodio u enfermedad actual tenía tres meses de evolución asociado con disnea, fiebre y síntomas constitucionales que se interpretaron como a un posible caso de neumonía por imagen y falla ventilatoria asociada. El manejo requirió intubación oro-traqueal en unidad de cuidados intensivos. Bajo intubación orotraqueal y soporte ventilatorio permaneció por 10 días y con antibióticos intravenosos. La extubación se realizó después del control de la infección respiratoria.

Posteriormente, al egreso, en la paciente persistía la disnea de moderados esfuerzos, la cual interpretaron como un posible caso de asma bronquial y se manejó con inhaladores. A pesar de cumplir con el tratamiento ambulatorio, la paciente no mostraba mejoría alguna, con persistencia de la disnea. Por el incremento de la misma con pocos minutos de caminar, se solicitó la consulta a Neumología. Entre los antecedentes relevantes estaban hipertrofia de cornetes y el uso de salmeterol/fluticasona polvo seco, ipratropiun y salbutamol inhalados durante los tres últimos meses.

Por la pandemia actual de COVID-19 la interconsulta se hizo por teleconsulta usando un programa de videollamada. En la primera parte de la teleconsulta se observó a una mujer en buen estado general, quien se mostraba alerta y orientada en persona, tiempo y lugar, y adecuada condición física global. La anamnesis y la observación de 
La teleconsulta especializada puede beneficiarse por la asistencia de un médico general, donde se cuenta con ese talento humano, porque en lugares remotos, con frecuencia, estos servicios están en manos de profesionales de enfermería. la paciente no eran suficientes para llegar a un diagnóstico y manejo apropiado. Se solicitó entonces la asistencia de un médico general, familiar de la paciente, quien completó el examen físico y encontró tensión arterial de 100/60, frecuencia cardíaca 140/min, frecuencia respiratoria de 28/min, saturación de oxígeno del $98 \%$ al aire y como hallazgos relevantes a la auscultación pulmonar informó la presencia de estridor laríngeo inspiratorio y espiratorio, sin roncus, ni sibilancias. Con esta información del examen físico, el neumólogo en teleconsulta pudo hacer un análisis más completo del caso.

La ausencia de ruidos respiratorios anormales a la auscultación cardiopulmonar y la presencia de estridor inspiratorio y espiratorio hicieron pensar en una posible enfermedad traqueal y se hizo la impresión clínica de un tumor traqueal. Las figuras 1A y 1B muestran exámenes complementarios solicitados y la confirmación de la impresión clínica. El análisis de patología informó tumor miofibroblástico inflamatorio.
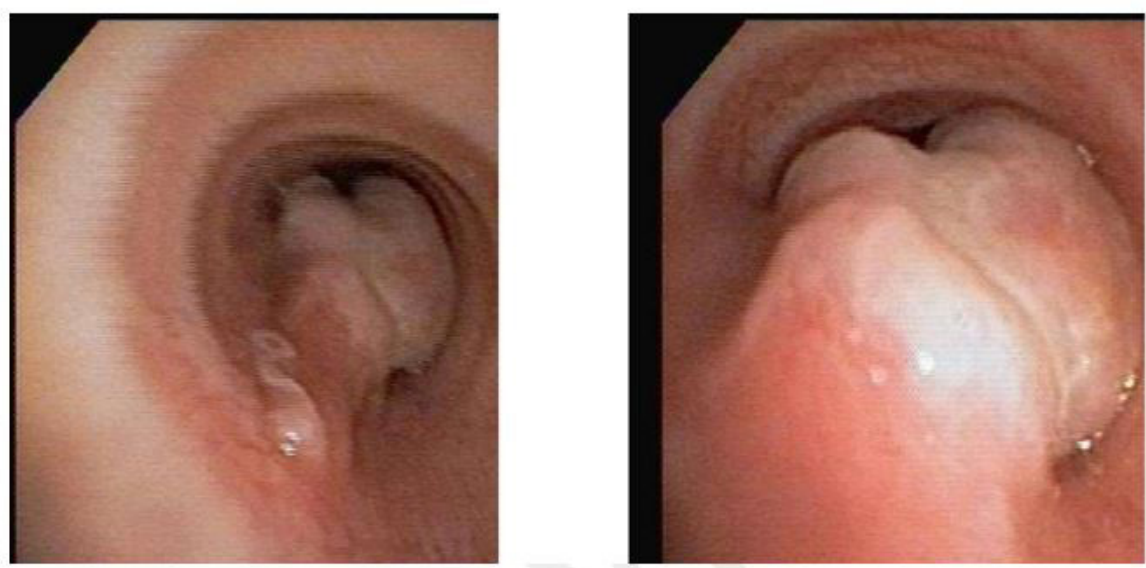

Figura 1A y 1B. Broncoscopia fibro-óptica que muestra masa en forma de pólipo adherida a la unión membranosa y cartilaginosa izquierda de la tráquea que disminuye la luz en $96 \%$ en el tercio inferior.

\section{Discusión}

Este caso ilustra una de las ventajas de la telemedicina al permite una evaluación de una paciente temerosa de una consulta presencial por el antecedente reciente de una complicación pulmonar. Al tiempo, muestra que la anamnesis se puede hacer por teleconsulta y da la relevancia el examen físico para el diagnóstico de un sinnúmero de condiciones clínicas (8).

Casos similares de enfermedad respiratoria manejados por teleconsulta no se han publicado durante la actual pandemia de enfermedad por coronavirus. No obstante, en India, Patra et al. informan la evaluación y manejo exitoso de dos casos de niños con comportamientos disruptivos evaluados por teleconsulta ante las restricciones en la movilidad (9). En Brasil, Accoris et al., presentan resultados satisfactorios de un caso de trombólisis guiada por teleconsulta a una paciente de 60 años con enfermedad por coronavirus quien presentó un accidente vascular cerebral isquémico, durante la estancia en un hospital de campo desplegado para la epidemia (10).

Posiblemente, la teleconsulta especializada puede beneficiarse por la asistencia de un médico general, donde se cuenta con ese talento humano, porque en lugares remotos, con frecuencia, estos servicios están en manos de profesionales de enfermería (11). 
Los usuarios se pueden beneficiar de la teleconsulta dado que brinda una consulta de fácil acceso, mejora la oportunidad, tiene alta capacidad resolutiva, diagnóstico y tratamiento adecuados y la seguridad de la información en las bases de datos. También permite a los servicios de salud mejorar la atención temprana, los diagnósticos oportunos y tratamientos adecuados, la reducción de los costos en la atención por la disminución de las urgencias y hospitalizaciones, impactar factores que afecten la morbimortalidad y, finalmente, realizar campañas preventivas de otras enfermedades concomitantes.
Además, se debe considerar que las personas más necesitadas de este tipo de servicios son aquellas que tienen menos acceso a la tecnología, por lo que se precisa de estrechar la brecha entre las áreas urbanas y rurales del país (12).

La telemedicina tiene muchos mitos para la implementación rutinaria: 1) menor calidad clínica que la consulta presencial; 2) requiere de alta tecnología; 3) es complicada la implementación; y 4) es muy costosa y poco rentable (13). En Colombia, la coyuntura de la pandemia de la COVID-19 ha permitido que se pueda mirar y avanzar hacia el uso y aplicación de esta estrategia (14). Se cuenta además con el marco legal que permite la implementación de la telemedicina y garantizar a todos los ciudadanos en acceso universal a la salud (15-17).

Los usuarios se pueden beneficiar de la teleconsulta dado que brinda una consulta de fácil acceso $(18,19)$, mejora la oportunidad (no filas, no salas de espera y menor costo) (20), tiene alta capacidad resolutiva, diagnóstico y tratamiento adecuados (21) y la seguridad de la información en las bases de datos (18). Por su parte, la teleconsulta permite a los servicios de salud mejorar la atención temprana, los diagnósticos oportunos y tratamientos adecuados, la reducción de los costos en la atención por la disminución de las urgencias y hospitalizaciones, impactar factores que afecten la morbimortalidad y, finalmente, realizar campañas preventivas de otras enfermedades concomitantes (22).

Se concluye que, en tiempo de epidemia y confinamiento, la teleconsulta especializada puede ser una herramienta útil en muchos casos para el diagnóstico de lesiones poco frecuentes. Posiblemente, se puede incrementar la eficacia diagnóstica de esta estrategia si se cuenta con la asistencia de un profesional médico que complete el examen físico en pacientes con síntomas agudos y, no simplemente, una consulta de seguimiento y control. El panorama para la teleconsulta y la telemedicina es prometedor si se consideran las limitaciones de acceso a los servicios de salud de un gran número de ciudadanos en el actual período de epidemia o en tiempos regulares.

\section{Consideraciones éticas}

La paciente consintió el uso de las imágenes diagnósticas del presente caso, después de informar que no se daría a conocer su nombre.

\section{Conflictos de intereses}

Los autores no tienen conflictos de interés que declarar.

\section{Fuente de financiación}

Universidad del Magdalena, Santa Marta, Colombia.

\section{Bibliografía}

1. Gilroy A, Lovett K. Incorporating telemedicine as part of COVID-19 outbreak response systems. Am J Manag Care. 2020;26(4):147-148. https://doi.org/10.37765/ ajmc.2020.42784.

2. Portnoy J, Waller M, Elliott T. Telemedicine in the era of COVID-19. J Allergy Clin Immunol Pract. 2020; 8:1489-1491. https://doi.org/10.1016/j.jaip.2020.03.008.

3. Hollander JE, Carr BG. Virtually Perfect? Telemedicine for Covid-19. N Engl J Med. 2020 30;382(18):1679-1681. https://doi.org/10.1056/NEJMp2003539. 
4. Khemapech I, Sansrimahachai W, Toachoodee M. Telemedicine-meaning, challenges and opportunities. Siriraj Med J. 2019;71(3):246-252. https://doi. org/10.33192/Smj.2019.38

5. Dheer A, Chaturvedi RK. Embracing a revolution-Telemedicine. Med J Armed Forces India. 2005;61(1):51-56. https://doi.org/10.1016/S0377-1237(05)80121-2

6. Chauhan V, Galwankar S, Arquilla B, Garg M, Di Somma S, El-Menyar A, et al. Novel coronavirus (COVID-19): Leveraging telemedicine to optimize care while minimizing exposures and viral transmission. J Emerg Trauma Shock. 2020;13(1):20-24. https:// doi.org/10.4103/JETS.JETS 3220

7. Pereira LJ, Pereira CV, Murata RM, Pardi V, Pereira-Dourado SM. Biological and social aspects of Coronavirus Disease 2019 (COVID-19) related to oral health. $\mathrm{Br}$ Oral Res. 2020;34(ePub May 08). https://doi.org/10.1590/1807-3107bor-2020. vol34.0041

8. Báez R, Monraz S, Castillo P, Rumbo U, García R, Ortíz R, et al. La exploración del tórax: una guía para descifrar sus mensajes. Rev Fac Med UNAM. 2016;59(6):4357. https://doi.org/10.1046/j.1365-2702.2003.00690.x

9. Patra S, Patro BK. COVID-19 and the need for child and adolescent telepsychiatry services, a case report. Asian J Psychiatry. 2020:54:102298. https://doi. org/10.1016/j.ajp.2020.102298

10. Accorsi TA, Brigido AR, Habrum FC, de Carvalho FA, Lima KDA, Pedrotti CH, et al. Telemedicine-guided stroke thrombolysis in a COVID-19 patient admitted in a field hospital. Arch Clin Med Case Report. 2020; 4(4):690-698. https://doi. org/10.26502/acmcr.96550252

11. Daly WM, Carnwell R. Nursing roles and levels of practice: a framework for differentiating between elementary, specialist and advancing nursing practice. J Clin Nurs. 2003;12(2):158-167.

12. Smith AC, Thomas E, Snoswell CL, Haydon H, Mehrotra A, Clemensen J, et al. Telehealth for global emergencies: Implications for coronavirus disease 2019 (COVID-19). J Telemed Telecare. 2020;26(5):309-313. https://doi.org/10.1177/1357633X20916567.

13. Correa AM. Avances y barreras de la telemedicina en Colombia. Rev Fac Der Cienc Polit UPB. 2017:47(127):361-381. http://dx.doi.org/10.18566/rfdcp.v47n127.a04

14. Márquez JR. Teleconsulta en la pandemia por Coronavirus: desafíos para la telemedicina pos-COVID-19. Rev Colomb Gastroenterol. 2020;35(1):5-16. https://doi. org/10.22516/25007440.543

15. Ley 1419 de 2010 por la cual se establecen los lineamientos para el desarrollo de la Telesalud en Colombia. Bogotá: Congreso de la República de Colombia; 2010.

16. Resolución 2654 del 2019 por la cual se establecen disposiciones para la telesalud y parámetros para la práctica de la telemedicina en el país. Bogotá: Ministerio de Salud y de la Protección social; 2019. 
17. Resolución 3100 del 2019 por la cual se definen los procedimientos y condiciones de inscripción de los prestadores de servicios de salud y de habilitación de los servicios de salud y se adopta el Manual de Inscripción de Prestadores y Habitación de Servicios de Salud. Bogotá: Ministerio de Salud y de la Protección social; 2019.

18. WebsterP.Virtual healthcare in the era ofCOVID-19.Lancet.2020;395(10231):11801181. https://doi.org/10.1016/S0140-6736(20)30818-7

19. Herreria J. America's Doctor Online provides easy access for consultations. Profiles Healthc Mark. 1999:15(1):31-32.

20. Almathami HKY, Win KT, Vlahu-Gjorgievska E. Barriers and facilitators that influence telemedicine-based, real-time, online consultation at patients' homes: systematic literature review. J Med Internet Res. 2020; 22(2):e16407. https://doi. org/10.2196/16407

21. Hong Z, Li N, Li D, Li J, Li B, Xiong W, et al. Telemedicine during the COVID-19 pandemic: experiences from Western China. J Med Internet Res. 2020;22(5):e19577. https://doi.org/10.2196/19577

22. Lupton D, Maslen S. Telemedicine and the senses: a review. Sociol Health Illness. 2017;39(8):1557-1571. https://doi.org/10.1111/1467-9566.12617 